\title{
The effect of gaseous accretion disk on dynamics of the stellar cluster in AGN
}

\author{
Bekdaulet Shukirgaliyev
}

Fesenkov Astrophysical Institute Observatory 23, 050020 Almaty, Kazakhstan email: bekdaulet@aphi.kz

\begin{abstract}
There is a supermassive black hole, a gaseous accretion disk and compact star cluster in the center of active galactic nuclei, as known today. So the activity of AGN can be represented as the result of interaction of these three subsystems. In this work we investigate the dynamical interaction of a central star cluster surrounding a supermassive black hole and a central accretion disk. The dissipative force acting on stars in the disk leads to an asymmetry in the phase space distribution of the central star cluster due to the rotating accretion disk. In our work we present some results of Stardisk model, where we see some changes in density and phase space of central star cluster due to influence of rotating gaseous accretion disk.
\end{abstract}

Keywords. galaxies: nuclei, galaxies: active, accretion, accretion discs, stellar dynamics

\section{Introduction}

Physical nature of AGN is far from full understanding because of big distances and relatively small sizes of energy-producing regions. Therefore development of AGN theory still remains one of the main problems in astrophysics (Beckmann \& Shrader 2013, Wu et al. 2015).

According to the dominant model, the phenomenon of AGN is explained by the accretion of matter onto a supermassive black hole in the center of galaxies (according to modern data (Kormendy \& Ho 2013), there are supermassive black holes with mass from a few billion to several trillions of solar masses in the center of most galaxies). In the process of accretion potential and kinetic energy of substances is effectively converted into radiation energy, which may explain the stable and very powerful radiation from a very small region, observed in AGNs. Since the angular momentum of accreting substances is retained, it forms a disk, and the system becomes axisymmetric.

However, there are typically spherically symmetric compact stellar clusters around central black hole in the central region of galaxies. The previous work (Just et al. 2012, Vilkoviskij et al. 2013) investigated how star-disk interactions effect the evolution of AGN using simpler model of accretion disk and numerical simulations. In particular, it was found that the dissipative star-disk interactions can significantly increase the rate of accretion of stars onto SMBH as stars transfer portion of the energy to gas and their orbits are stacked in the plane of accretion disk, and as a result they also accreting onto the central black hole (Just et al. 2012). However, to assess the impact of the accretion disk in the orbital and phase characteristics of the stars, one needs to explore a more realistic model of the disk.

The investigated AGN model includes three subsystems: a compact star cluster (CSC), the accretion disk (AD), and the central supermassive black hole (SMBH). A star cluster is simulated by direct integration of the individual stars interaction with each other (direct $N$-body simulations) and with a gas disk and black hole. Gaseous accretion disk is defined phenomenologically with density distribution constant in time, and has a 
Keplerian rotation curve. Black hole is also defined phenomenologically as a Newtonian potential. If the particle comes inside the region with radius less than $R_{a c c r}$ (radius of accretion), then we consider that it is accreted onto the supermassive black hole. Once it happened, the particle disappears and its mass is added to the SMBH. We used modified version of phiGRAPE code (Harfst et al. 2007)for our simulations, which uses parallel computing technology of NVIDIA CUDA and MPI. The stellar component of the system is defined as a Plummer density profile initially. A more detailed description of the numerical model can be found in Just et al. 2012, Vilkoviskij et al. 2013.

In Kazakhstan the first computer cluster specialized for $N$-body simulations was created in 2008 within the frame of the joint Kazakh-German "STARDISC" project which combined forces of groups in Heidelberg University and Fesenkov Astrophysical Institute. This work presents results of investigations of evolution and physics problems in AGN are calculated in this computer cluster as well as in computer cluster of Astronomisches Rechen-Institute in Germany.

\section{Accretion disk model}

Let us consider the model of the accretion disk. We take as a basis a three-dimensional, axisymmetric stationary disk, which is characterized by differential rotation with the local angular velocity. The radial profile of the surface density is specified as

$$
\Sigma(R)=\Sigma_{d}\left(\frac{R}{R_{d}}\right)^{-\alpha},
$$

here $\alpha=\frac{3}{4}, R^{2}=x^{2}+y^{2}, R_{d}$ is disk radius and $\Sigma_{d}$ is surface density value at $R=R_{d}$. The value $\alpha=3 / 4$ corresponds to the outer boundary of the disk model of the Novikov \& Thorne 1973. Disk mass is equal to

$$
M_{d}=2 \pi \int_{0}^{R_{d}} \Sigma(R) R d R=\frac{2 \pi}{2-\alpha} \Sigma_{d} R_{d}^{2} .
$$

For the numerical integration of the equations of motion the force acting on the particle should be smooth and continuous function, so one needs enter the exponential factor that ensures a smooth (but fast enough) decreasing of gas density at the edge of the disk (Just et al. 2012).

$$
\Sigma(R)=\Sigma_{d}\left(\frac{R}{R_{d}}\right)^{-\alpha} e^{-\beta_{s}\left(\frac{R}{R_{d}}\right)^{s}} .
$$

For the resulting expression to correspond to the equation 2.2 we selected

$$
\beta_{s}=\Gamma\left(1+\frac{2-\alpha}{s}\right),
$$

wherein $\Gamma(x)$ - the gamma function. Let us take $s=4$, then beta $a_{s}=0.70$ for $\alpha=3 / 4$. In this case, if $R=R_{d}$ the surface density is equal to $\Sigma\left(R_{d}\right)=0.49 \Sigma_{d}$ (Just et al. 2012). To numerically model the described model we select isothermal density profile, defined as

$$
\rho_{g}(R, z)=\frac{\Sigma(R)}{\sqrt{2 \pi} h_{z}} \exp \left(-\frac{z^{2}}{2 h_{z}^{2}}\right) .
$$

Previous works (Just et al. 2012, Vilkoviskij et al. 2013) used constant thickness accretion disk model

$$
h_{z}=h R_{d}
$$




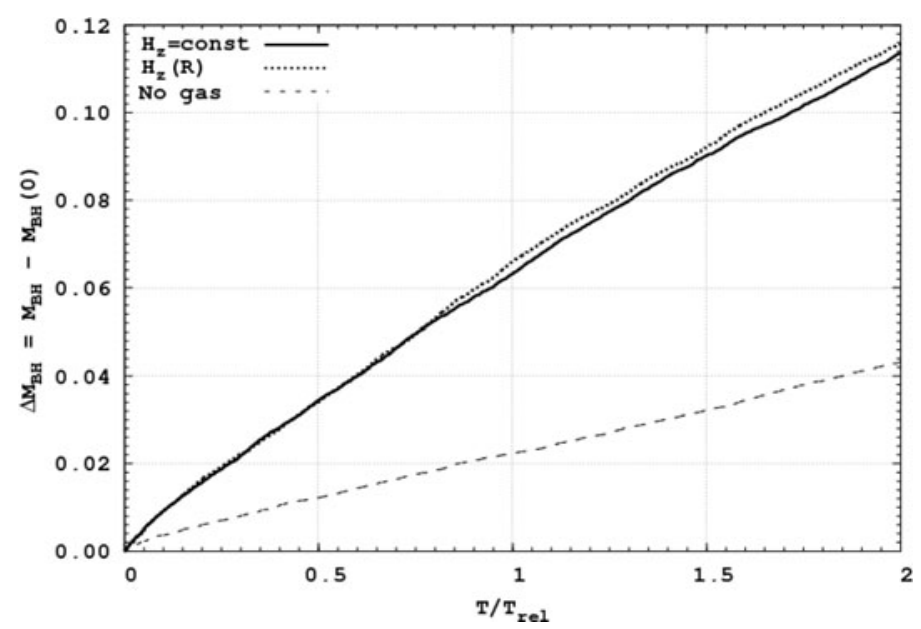

Figure 1. The black hole mass growth as a function of time for three model runs - the old model of disk (solid line), with the new model (dotted line) and a control run without gas disk (dotted line). The mass of black hole is in dimensionless $N$-body units, the unit of time specified in the relaxation time of the system.

If the relationship 2.5 is substituted into the expression 2.4 , we obtain the expression for the density of disk with a constant height:

$$
\rho(R, z)=\frac{2-\alpha}{2 \pi \sqrt{2 \pi}} \frac{M_{d}}{h R_{d}^{3}}\left(\frac{R}{R_{d}}\right)^{-\alpha} e^{-\beta_{s}\left(\frac{R}{R_{d}}\right)^{s}} e^{-\frac{z^{2}}{2 h^{2} R_{d}^{2}}} .
$$

In this work we consider a new model, which is a modification of the first model of accretion disk with the introduction of a linear function to increase the disk half thickness in the inner part. This modification is based on the physical properties of the inner accretion disk, which is described by the approach of Shakura \& Sunyaev 1973

$$
h_{z}=h R_{d}\left(\frac{R}{R_{\text {crit }}}\right) .
$$

The transition point from linearly increasing to constant thickness is in the point where the disk is vertically self-gravitating at $R=R_{\text {crit }}$. In our simulations $R_{\text {crit }}=0.0257314$ in $N$-body dimensionless unit system (Hénon 1971).

The properties of the accretion disc are fixed by the reduced mass with analytical density distribution according to equation 2.6 with the values $\alpha=3 / 4, s=4$ and $h=10^{-3}$. The disk has Keplerian rotation in the potential of SMBH neglecting the gravitational influence of the disk and pressure gradients within the disk (Just et al. 2012).

\section{Results}

To ensure that the new model of disk does not change the global dynamics of the system and thereby does not contradict the results we obtained earlier, we compared the black hole mass growth rate due to the accretion of stars (Fig. 1).

As can be seen in Fig. 1 the evolution of the black hole during two relaxation times for both accretion disk models is identical. At the same graph control run without gaseous disk is shown. In this case, the black hole mass growth is due to only the capture of those stars, which accidentally happened to be within the accretion radius. We also investigate the impact of accretion disk on the dynamical characteristics of stellar cluster. In 


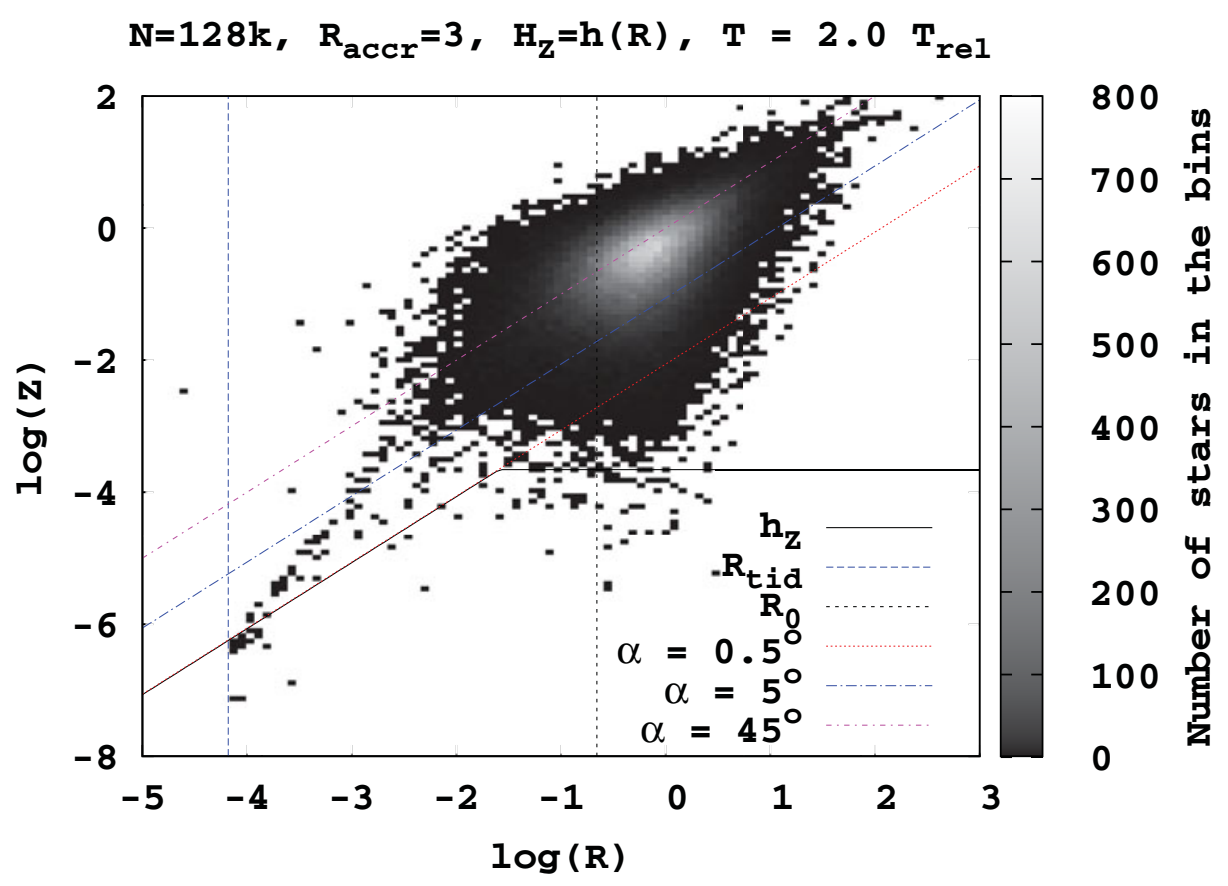

Figure 2. Stellar distribution in cylindrical coordinate system. Here stellar disk formed in the inner part of the system shown as a tail to the center of the system.

particular, we analysed orbital parameters of accreted particles. We found that these two disk models give different results in orbital parameters distribution of captured particles. Specifically, the new disk model allows stars to survive in the accretion disk for longer times than in old disk model. So we get much more particles accreted in near-circular orbits $(e \approx 0)$ in the disk plane $\left(i<0.5^{\circ}\right)$. Although both disk models initiate the same growth rate of the central SMBH (Fig.1), the gaseous disk with a constant thickness captures more counter-rotating stars, in contrast to the disk with a variable thickness.

If we decrease the accretion radius value in the previous model of the disk, the gas density increases in the central part, but its thickness remains constant and it rotates around the center on Keplerian orbits. This leads to deceleration of many stars on orbits counter-rotating with the disk in the inner part of the system, including stars in nearly perpendicular orbits to the disk plane. In case of an improved disk model, super-dense gas occurs practically only in the equatorial plane near the central black hole, and that allows many stars in the central part to evolve towards the direction of rotation of the disk. Stars evolved in the positive direction of rotation relatively to the direction of the disk rotation could increase the contribution of the positive Z-component of the angular momentum in the central part of the cluster.

We found that the disk model with varying thickness leads to formation of relatively stable disk from stars in the inner part of the system. It captures stars which are going to be accreted and allows them to live longer in the disk. One can see formation of such disk in (Fig. 2). The central star cluster changes its structure in the inner part and forms stellar disk in the early stage of simulation $\left(T \approx 0.01 T_{\text {rel }}\right)$. This structure of star cluster retained during two relaxation times (up to the end of the simulations).

Thus, we see that the resulting phenomenological model of gas accretion disk is physically adequate and the studied density profile can be used in the future, including 
modeling of the gas disk directly using the methods of hydrodynamics. In the future, we plan to improve the numerical model of AGNs in order to allow additional processes (collisions of stars in the central part, stellar evolution, primordial binaries, binary black holes, etc.), and to perform one-to-one simulations, i.e. with the number of stars equal to that in real clusters (about one million stars).

\section{Acknowledgements}

I would like to thank my collaborators G. Kennedy, Y. Meiron, P. Berczik, A. Just, T. Panamarev, M. Makukov, D. Yurin, C. Omarov, E. Vilkoviski, and R. Spurzem. We will publish a more detailed presentation of results elsewhere. I also thank International Astronomical Union for its grant which gave me this opportunity to participate in IAU Symposium No. 312 in Beijing in 2014.

\section{References}

Beckmann, V. \& Shrader, C. 2013, Active galactic nuclei (John Wiley \& Sons.), 374 p.

Harfst, S., Gualandris, A., Merritt, D., Spurzem, R., Zwart, S. P., \& Berczik, P. 2007, New Astronomy, 12, 357

Hénon, M 1971, Astrophys. Space Sci., 14, 151

Just, A., Yurin, D., Makukov, M., Berczik, P., Omarov, Ch., Spurzem, R., \& Vilkoviskij, E. Y. 2012, ApJ, 758, 51

Kormendy J. \& Ho L. C. 2013, Annu. Rev. Astron. Astrophys, 51, 511

Novikov I. D., Thorne K. S. 1973, in Dewitt C., Dewitt B. S., eds, Black Holes (Les Astres Occlus) Astrophysics of black holes.., pp 343-450

Shakura N. I. \& Sunyaev R. A. 1973, A\&A, 24, 337

Vilkoviskij E., Makukov M., Omarov Ch., Panamarev T., Spurzem R., Berczik P., \& Just A. 2013, A\&AT, 28, 151

Wu X.-B., Wang F., Fan X., Yi W., Zuo W., Bian F., Jiang L., McGreer I. D., Wang R., Yang J., Yang Q., Thompson D., \& Beletsky Y 2015, Nature, 518, 512 